\title{
The therapeutic effect of splenectomy plus Meta-Analysis pericardial devascularization versus conventional pericardial devascularization on portal hypertension in China: a meta-analysis
}

\author{
Yajie Zhao ${ }^{1}$ and Chengfeng Wang ${ }^{1}$ \\ ${ }^{1}$ Department of Pancreatic and Gastric Surgery, National Cancer Center/Cancer Hospital, Chinese Academy of Medical \\ Sciences and Peking Union Medical College, Beijing 100021, China \\ Correspondence to: Chengfeng Wang, email: zhaoyajielancet@163.com \\ Keywords: selective esophagogastric devascularization; non-selective esophagogastric devascularization; portal hypertension; \\ meta-analysis \\ Received: November 29, 2017 Accepted: December 26, 2017 Epub: January 03, 2018 Published: March 16, 2018 \\ Copyright: Zhao et al. This is an open-access article distributed under the terms of the Creative Commons Attribution License 3.0 \\ (CC BY 3.0), which permits unrestricted use, distribution, and reproduction in any medium, provided the original author and source \\ are credited.
}

\section{ABSTRACT}

Background: To systematically review perioperative outcomes and postoperative complications between splenectomy plus s-EGDV and n-sEGDV for portal hypertension complicated with thoracic esophageal varices and bleeding by a meta-analysis.

Method: We searched the databases of PubMed, the Cochrane Library, Web of Science, EMBASE, TCGA, Chinese Biomedicine Database from January 2000 to June 2017, and included studies that compared perioperative outcomes and postoperative complications between s-EGDV and n-sEGDV. These included studies were assessed by two independent investigators.

Results: Seven randomized controlled trials (RCTs) and seven non-randomized observational clinical studies (OCS) were included. The S-EGDV was more beneficial than $n-s E G D V$ in reducing the PVF $\left(O R=4.26 ; 95 \% C I, 2.81-5.71 ; P<0.00001 ; I^{2}=\right.$ 97\% for heterogeneity), portal vein flow (OR $=-111.75 ; 95 \% C I,-197.13-26.38 ; P=$ $0.01 ; I^{2}=90 \%$ for heterogeneity), portal hypertensive gastropathy( $O R=0.38 ; 95 \%$ CI, 0.28-0.51; $P<0.00001 ; I^{2}=0 \%$ for heterogeneity), hepatic encephalopathy (OR $=0.40 ; 95 \% \mathrm{CI}, 0.23-0.71 ; P=0.002 ; I^{2}=22 \%$ for heterogeneity), postoperative re-bleeding (OR $=0.43 ; 95 \% \mathrm{CI}, 0.29-0.63 ; P<0.0001 ; I^{2}=9 \%$ for heterogeneity), postoperative mortality $\left(O R=0.52 ; 95 \% C I, 0.32-0.85 ; P=0.009 ; I^{2}=0 \%\right.$ for heterogeneity) and in increasing hepatic artery flow (OR $=92.53 ; 95 \%$ CI, 9.60175.46; $P=0.03 ; I^{2}=95 \%$ for heterogeneity).

Conclusion: sEGDV offers a more effective surgical approach with fewer complications to treat portal hypertension than n-sEGDV. Upon further detailed analysis of the surgical indications and hemodynamic and postoperative major complications of selective devascularization, sEGDV likely will provide us with a new direction in the choice of surgical approach for portal hypertension.

\section{INTRODUCTION}

In China, cases of hepatitis leading to liver cirrhosis and portal hypertension are on the rise. The typical clinical manifestations of portal hypertension are splenomegaly and hypersplenism, portacaval collateral, and ascites; in some patients, this is further complicated by esophageal varices and bleeding, ultimately resulting in mortality [1]. Portosystemic shunt (PSS) and gastroesophageal devascularization (GD) are the main surgical treatment methods [2]. PSS operation can significantly reduce portal venous pressure, and the rate of bleeding can be controlled by $85 \%-100 \%$. Further, PSS can not only eliminate ascites in a rapid and efficient manner but also 
improve blood circulation of the gastric mucosa [3-4]. However, PSS does not conform to normal physiology. It reduces the blood flow from the portal vein to the liver and even causes blood outflow from the liver, resulting in further liver dysfunction after operation [5]. The incidence of hepatic encephalopathy is high, and the operation is very traumatic. Devascularization is a surgical procedure to reduce or block the portal blood flow and the communicating veins of the portal vein and the azygos vein system. In 1929, Walter first reported ligation of the gastric coronary vein, following which several types of devascularization procedures were followed. Among them, splenectomy plus esophagogastric devascularization (EGDV) is the most effective [6]; hence, it has become the first treatment choice in portal hypertension complicated with thoracic esophageal varices and bleeding, in China. In terms of clinical research development, especially the anatomical study of the lower esophagus and cardia, selective esophagogastric devascularization (s-EGDV) has gained more popularity. s-EGDV is an improved surgical method based on traditional pericardial devascularization; s-EGDV only devascularizes the branch veins that enter the esophagus and stomach wall. Compared with traditional pericardial devascularization, s-EGDV not only devascularizes the branch veins to prevent bleeding but also retains the integrity of the gastric coronary vein and paraesophageal veins and maintains spontaneous portacaval shunt. The selective process guarantees spontaneous shunt of the body's part of the portal vein and ensures the direction of blood flow from the gastric coronary vein to the paraesophageal veins to the semiazygos vein. Portoazygous spontaneously shunt, which forms a shunt between the portal and azygos vein, offers a compensatory mechanism, as the resulting blood flow is reasonable, appropriate, and physiologically compatible. It can maintain the necessary hepatic blood flow and appropriately reduce portal vein pressure to achieve a dynamic balance. It is different from the manmade spleen-renal shunt or portal vein-vena cava shunt, this spontaneously shunt should be retained. s-EGDV can preserve this spontaneous shunt on the basis of devascularization, which can achieve combined operation of the shunt and devascularization. Some randomized controlled trials (RCTs) and observational clinical studies (OCSs) were conducted to address this issue. Several published studies have shown convincing results in recent years. Therefore, this meta-analysis aimed to compare the perioperative outcomes and postoperative complications between s-EGDV and n-sEGDV.

\section{MATERIALS AND METHODS}

\section{Literature search}

We searched the databases of the Cochrane Library, PubMed, EMBASE, web of Science, TCGA and the
Chinese Biomedicine Database from January 2000 to June 2017 both electronically and manually following search terms: 'selective esophagogastric devascularization' OR 's-EGDV' OR 'selective pericardial devascularization' And 'esophagogastric devascularization' OR 'EGDV' OR 'pericardial devascularization' And 'Non-Selective esophagogastric devascularization' OR 'n-sEGDV' OR 'conventional pericardial devascularization' And 'portal hypertension'. Both MeSH words and free terms were included in the search, No language restriction and two independent researchers performed this search. Final inclusion was determined by consensus. The results of the search strategy are shown in Table 1.

\section{Inclusion criteria}

The following studies were included: (1) those that compared the perioperative outcomes and postoperative complications between s-EGDV and n-sEGDV for cirrhotic patients with portal hypertension complicated with thoracic esophageal varices and bleeding; and (2) those that were the most recent publication in the case of multiple similar reports.

\section{Exclusion criteria}

The following studies were excluded: (1) those wherein the detailed surgical type was not reported; (2) those with no comparison of s-EGDV and n-sEGDV; (3) those in which the study outcomes did not include complete or available perioperative outcomes and postoperative data; (4) those which reported data used in a later study; and (5) case reports, abstracts, letters, comments, reviews, guideline articles without original data, and studies that presented insufficient data.

\section{Data extraction}

The following detailed data were extracted by two independent investigators: authors; year of publication; country; study design; surgery type; number of patients; and the following clinical data: (1) The decrement of FPP ( $\mathrm{cm} \mathrm{H}_{2} \mathrm{O}$ ): select the right gastroepiploic vein catheter to portal vein, the zero point in axillary midline, the mean value of 3 time as the value of preoperative FPP, stop operation to complete $10 \mathrm{~min}$ measured again after 3 times, the average value as the FPP value after operation. The difference between the two is FPP reduction. (2) The value of PVF ( $\mathrm{mL} / \mathrm{min})$ : The portal vein diameter (D) and maximum flow velocity (Vmax) were measured by ultrasound before and three months after surgery. Then, the value of PVF was calculated according to the formula $\mathrm{Q}=\pi / 4 \mathrm{D} \times 0.57 \mathrm{Vmax} \times 60$. (3) Hepatic artery flow (HAF): The hepatic artery diameter (D) and maximum flow velocity (Vmax) were measured by ultrasound before and three months after surgery. Then, the value of HAF was calculated according to the formula $\mathrm{Q}=\pi / 4 \mathrm{D} \times 0.57$ 
Table 1: Flow diagram

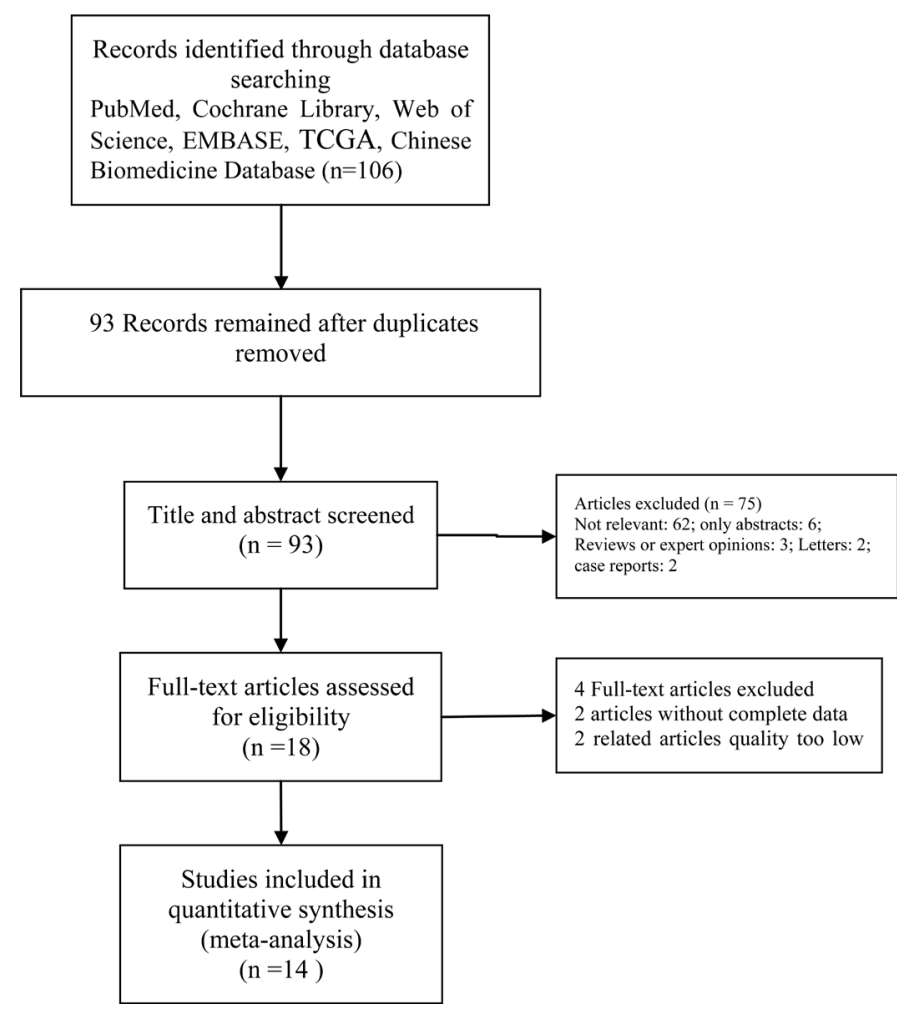

Vmax $\times 60$. (4) Portal hypertensive gastropathy (PHG), (5) hepatic encephalopathy, (6) postoperative re-bleeding rate; (7) postoperative mortality.

\section{Statistical analysis}

Meta-analysis was conducted with Review Manager (version 5.3.0) software. Odds ratios (ORs) were used to analysis the dichotomous variables and $95 \%$ confidence interval (CI) values were reported. The Mantel-Haenszel, Chi-square, and $\mathrm{I}^{2}$ tests were used to test the heterogeneity between studies. $I^{2}>50 \%$, this suggested significant heterogeneity, a random effects model was applied. If $\mathrm{I}^{2}<$ $50 \%$, this suggested not significant heterogeneity, a fixed effects model was applied. If $P<0.05$, this considered statistically significant. Funnel plots were used to evaluate potential publication bias.

\section{Characteristics of the included studies and quality assessment}

14 studies (seven randomized clinical trials RCTs and seven retrospective cohort studies) were included in this meta-analysis. The total number of patients was 1637 , of whom 766 was s-EGDV group and 871 was n-sEGDV group. The detailed characteristics of all the included studies are shown in Table 2.

\section{Assessment of the risk of bias of RCTs}

For the included RCTs, assessment of the bias risk involved six parameters: allocation concealment, incomplete outcome data, blinding, selective reporting bias, sequence generation, and other potential sources of bias. Assessment was based on a quality checklist recommended in the Cochrane Handbook. "Yes" indicated a "low" risk of bias; "unclear," an "uncertain" risk of bias; "no," a "high" risk of bias (Figure 1).

\section{META-ANALYSIS RESULTS}

\section{The reduction of PVF}

Eight included studies reported the reduction of PVF, we pooled data from the eight studies to comparing n-sEGDV group with sEGDV group. The results of meta-analysis indicate that there is significant difference between two groups in the reduction of PVF $(\mathrm{OR}=$ 4.26; 95\% CI, 2.81-5.71; $P<0.00001 ; \mathrm{I}^{2}=97 \%$ for heterogeneity), Therefore, using a Random model. The meta-analysis of RCTs and OCS subgroup both reveals a statically different between two groups in the reduction of PVF. [RCTs $\left(\mathrm{I}^{2}=98 \%, \mathrm{OR}=8.04 ; 95 \%\right.$ CI, 2.07-14.01; $P=0.008)$, OCS $\left(\mathrm{I}^{2}=88 \%\right.$, OR $=3.08 ; 95 \% \mathrm{CI}, 2.23-$ 3.92; $P<0.00001)]$ (Figure 2). 
Table 2: The characteristics of all the included studies

\begin{tabular}{|c|c|c|c|c|c|c|c|c|}
\hline Author & year & Country & Study type & Group & $\begin{array}{l}\text { Patients } \\
\text { number }\end{array}$ & $\begin{array}{c}\text { Male/ } \\
\text { female }\end{array}$ & Age, y & $\begin{array}{c}\text { Study quality } \\
\text { RCT (jadad system) } \\
\text { retro (NOS system) }\end{array}$ \\
\hline \multirow[t]{2}{*}{ Zhao B et al. [7] } & 2016 & China & RCT & s-EGDV & 40 & - & - & 5 \\
\hline & & & & n-sEGDV & 40 & - & - & \\
\hline \multirow[t]{2}{*}{ Zhang SJ et al. [8] } & 2014 & China & $\mathrm{RCT}$ & s-EGDV & 58 & $32 / 26$ & $48.12 \pm 9.34$ & 7 \\
\hline & & & & n-sEGDV & 58 & $31 / 27$ & $49.08 \pm 9.21$ & \\
\hline \multirow[t]{2}{*}{ Wang C et al. [9] } & 2014 & China & $\mathrm{RCT}$ & s-EGDV & 90 & $40 / 50$ & $47 \pm 13$ & 7 \\
\hline & & & & n-sEGDV & 90 & $45 / 45$ & $43 \pm 11$ & \\
\hline \multirow[t]{2}{*}{ Gu GJ et al. [10] } & 2013 & China & $\mathrm{RCT}$ & s-EGDV & 30 & $21 / 9$ & $45.7 \pm 4.7$ & 5 \\
\hline & & & & n-sEGDV & 30 & $23 / 7$ & $45.9 \pm 4.5$ & \\
\hline \multirow[t]{2}{*}{ Gong QH et al. [11] } & 2013 & China & RCT & s-EGDV & 93 & $67 / 26$ & $41.3 \pm 7.5$ & 7 \\
\hline & & & & n-sEGDV & 93 & $68 / 25$ & $41.7 \pm 6.9$ & \\
\hline \multirow[t]{2}{*}{ Wang WS et al. [12] } & 2012 & China & $\mathrm{RCT}$ & s-EGDV & 52 & - & - & 7 \\
\hline & & & & n-sEGDV & 50 & - & - & \\
\hline \multirow[t]{2}{*}{ Pan WN et al. [13] } & 2009 & China & $\mathrm{RCT}$ & s-EGDV & 91 & $59 / 32$ & $47(32-69)$ & 7 \\
\hline & & & & n-sEGDV & 85 & $55 / 30$ & $48(27-71)$ & \\
\hline \multirow[t]{2}{*}{ Zou SH et al. [14] } & 2017 & China & OCS & s-EGDV & 32 & & & 5 \\
\hline & & & & n-sEGDV & 30 & & & \\
\hline \multirow[t]{2}{*}{ Zhang Y et al. [15] } & 2016 & China & OCS & s-EGDV & 55 & $34 / 21$ & $45 \pm 17$ & 5 \\
\hline & & & & n-sEGDV & 89 & $49 / 40$ & $43 \pm 15$ & \\
\hline \multirow[t]{2}{*}{ Liu GF et al. [16] } & 2013 & China & OCS & s-EGDV & 48 & $28 / 20$ & $\begin{array}{c}58.26 \pm \\
10.29\end{array}$ & 7 \\
\hline & & & & n-sEGDV & 48 & $27 / 21$ & $\begin{array}{c}57.75 \pm \\
11.16\end{array}$ & \\
\hline \multirow[t]{2}{*}{ Ren DF etal. [17] } & 2013 & China & OCS & s-EGDV & 45 & $33 / 12$ & 42 & 5 \\
\hline & & & & n-sEGDV & 41 & $30 / 11$ & 45 & \\
\hline \multirow[t]{2}{*}{ You DY et al. [18] } & 2013 & China & OCS & s-EGDV & 28 & - & - & 5 \\
\hline & & & & n-sEGDV & 62 & - & - & \\
\hline \multirow[t]{2}{*}{ Zhao H et al. [19] } & 2011 & China & OCS & s-EGDV & 57 & $35 / 22$ & $42 \pm 16$ & 5 \\
\hline & & & & n-sEGDV & 51 & $30 / 21$ & $43 \pm 12$ & \\
\hline \multirow[t]{2}{*}{ Cen J et al. [20] } & 2005 & China & OCS & s-EGDV & 47 & $35 / 12$ & $44.6(21-72)$ & 7 \\
\hline & & & & n-sEGDV & 104 & $76 / 28$ & $43.5(26-67)$ & \\
\hline
\end{tabular}

RCT = randomized controlled trial; OCS: retrospective cohort studies. Jadad scale system: The Jadad scale, sometimes known as Jadad scoring or the Oxford quality scoring system, is a procedure to independently assess the methodological quality of a clinical trial. It is the most widely used such assessment in the world; The system was used to assess randomization, concealment of allocation, blinding, and withdrawals in the study. Each item was given a score of 0-2 and 7 score in total. If the total score was $\geq 4$, the RCT was of high quality. The Newcastle-Ottawa System: The quality of the nonrandomized studies was assessed by using this System, The quality of the studies was evaluated by examining three items: patient selection, comparability of groups, and assessment of outcome. Each study was given 9 score in total; if the total score was $\geq 7$, the OCS was considered to be of high quality

\section{Portal vein flow}

Three included studies reported the portal vein flow. The results of meta-analysis show that there is no difference between two groups in the portal vein flow $\left(\mathrm{OR}=-111.75 ; 95 \% \mathrm{CI},-197.13-26.38 ; P=0.01 ; I^{2}\right.$ $=90 \%$ for heterogeneity), Therefore, using a Random model (Figure 3).

\section{Hepatic artery flow}

Three included studies reported the hepatic artery flow. The results of meta-analysis show that there is no difference between two groups in the hepatic artery flow $(\mathrm{OR}=92.53 ; 95 \% \mathrm{CI}, 9.60-175.46$; $P=0.03 ; \mathrm{I}^{2}=95 \%$ for heterogeneity), Therefore, using a Random model (Figure 4). 


\section{Portal hypertensive gastropathy}

Nine included studies reported the portal hypertensive gastropathy. The results of meta-analysis show that there is difference between two groups in the portal hypertensive gastropathy $(\mathrm{OR}=0.38 ; 95 \% \mathrm{CI}$, $0.28-0.51 ; P<0.00001 ; I^{2}=0 \%$ for heterogeneity), Therefore, using a fixed model. The meta-analysis of RCTs and OCS subgroup both reveals a statically different between two groups in the portal hypertensive gastropathy. [RCTs $\left(I^{2}=53 \%\right.$, OR $=0.36 ; 95 \%$ CI, $0.24-0.53$;
$P<0.00001)$, OCS $\left(\mathrm{I}^{2}=0 \%, \mathrm{OR}=0.40 ; 95 \% \mathrm{CI}, 0.24-\right.$ $0.65 ; P=0.0003)]$ (Figure 5).

\section{Hepatic encephalopathy}

Ten included studies reported the incidence of hepatic encephalopathy. The results of meta-analysis show that there is no significant difference between two groups in the incidence of hepatic encephalopathy $\left(\mathrm{OR}=0.40 ; 95 \%\right.$ CI, $0.23-0.71 ; P=0.002 ; I^{2}=22 \%$ for heterogeneity), Therefore, using a fixed model. The

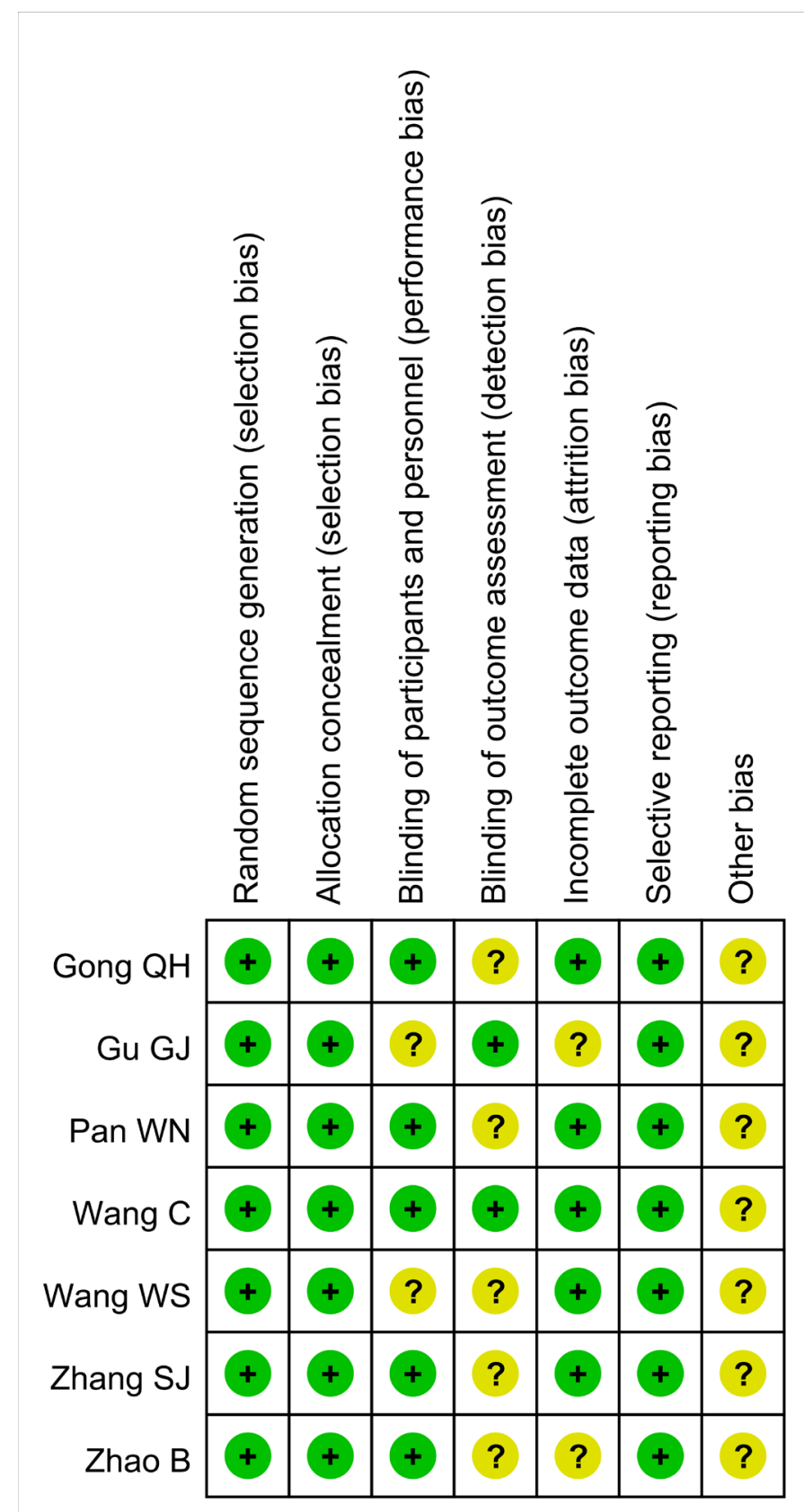

Figure 1: Risk of bias of RCTs: Assessment was based on a quality checklist recommended in the Cochrane Handbook. "Yes" indicated a "low" risk of bias; "unclear," an "uncertain" risk of bias; "no," a "high" risk of bias. 
meta-analysis of RCTs and OCS subgroup both reveals a statically different between two groups in the incidence of hepatic encephalopathy. [RCTs $\left(I^{2}=31 \%, \mathrm{OR}=0.31 ; 95 \%\right.$ CI, $0.15-0.66 ; P=0.002), \mathrm{OCS}\left(I^{2}=8 \%, \mathrm{OR}=0.57 ; 95 \%\right.$ CI, 0.24-1.33; $P=0.019)$ ] (Figure 6).

\section{Postoperative re-bleeding}

Thirteen included studies reported the incidence of postoperative re-bleeding. The results of meta- analysis show that there is significant difference between two groups in the incidence of postoperative re-bleeding $(\mathrm{OR}=0.43 ; 95 \% \mathrm{CI}, 0.29-0.63 ; P<$ $0.0001 ; I^{2}=9 \%$ for heterogeneity), Therefore, using a fixed model. The meta-analysis of RCTs and OCS subgroup both reveals a statically different between two groups in the incidence of postoperative re-bleeding. [RCTs $\left(I^{2}=0 \%, \mathrm{OR}=0.37 ; 95 \% \mathrm{CI}, 0.23-0.60 ; P<\right.$ $0.0001)$, OCS $\left(I^{2}=34 \%\right.$, OR $=0.17 ; 95 \%$ CI, $0.29-$ $1.01 ; P=0.05)$ ] (Figure 7).

\begin{tabular}{|c|c|c|c|c|c|c|c|c|c|c|c|c|c|}
\hline \multirow[b]{2}{*}{ Study or Subgroup } & \multicolumn{3}{|c|}{ sEGDV } & \multicolumn{3}{|c|}{ n-sEGDV } & \multicolumn{3}{|c|}{ Mean Difference } & \multirow{2}{*}{\multicolumn{4}{|c|}{$\begin{array}{l}\text { Mean Difference } \\
\text { IV. Random, } 95 \% \mathrm{Cl}\end{array}$}} \\
\hline & Mean & SD & Total & Mean & SD & Total & Weight & IV. Random, $95 \% \mathrm{CI}$ & Year & & & & \\
\hline \multicolumn{14}{|l|}{ 2.1.1 RCT } \\
\hline Wang C & 19.9 & 2.97 & 90 & 8.81 & 4.73 & 90 & $12.3 \%$ & $11.09[9.94,12.24]$ & 2014 & & & & 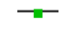 \\
\hline Wang WS & 16.1 & 2.4 & 52 & 11.1 & 3.1 & 50 & $12.4 \%$ & $5.00[3.92,6.08]$ & 2012 & & & & \\
\hline Subtotal $(95 \% \mathrm{Cl})$ & & & 142 & & & 140 & $24.8 \%$ & $8.04[2.07,14.01]$ & & & & & \\
\hline \multicolumn{14}{|c|}{$\begin{array}{l}\text { Heterogeneity: } \mathrm{Tau}^{2}=18.22 ; \mathrm{Chi}^{2}=57.10, \mathrm{df}=1(\mathrm{P}<0.00001) ; \mathrm{I}^{2}=98 \% \\
\text { Test for overall effect: } \mathrm{Z}=2.64(\mathrm{P}=0.008)\end{array}$} \\
\hline \multicolumn{14}{|l|}{$2.1 .2 \mathrm{cos}$} \\
\hline Zou SH & 9.8 & 4.1 & 32 & 7.3 & 4 & 30 & $10.6 \%$ & $2.50[0.48,4.52]$ & 2017 & & & & \\
\hline Zhang $Y$ & 15.9 & 2.7 & 55 & 14.2 & 3.1 & 89 & $12.6 \%$ & $1.70[0.74,2.66]$ & 2016 & & & - & \\
\hline Ren DF & 7.42 & 1.74 & 45 & 4.45 & 1.92 & 41 & $12.9 \%$ & $2.97[2.19,3.75]$ & 2013 & & & & \\
\hline Zhao H & 14.8 & 2.5 & 57 & 11.2 & 2.1 & 51 & $12.8 \%$ & $3.60[2.73,4.47]$ & 2011 & & & & \\
\hline You DY & 10.89 & 1.03 & 24 & 8.05 & 1.02 & 50 & $13.2 \%$ & $2.84[2.34,3.34]$ & 2007 & & & 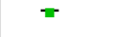 & \\
\hline Cen J & 10.49 & 1.05 & 39 & 6.13 & 0.99 & 84 & $13.2 \%$ & $4.36[3.97,4.75]$ & 2005 & & & & \\
\hline Subtotal $(95 \% \mathrm{Cl})$ & & & 252 & & & 345 & $75.2 \%$ & $3.08[2.23,3.92]$ & & & & & \\
\hline \multicolumn{14}{|c|}{ Heterogeneity: Tau $^{2}=0.90 ; \mathrm{Chi}^{2}=41.47, \mathrm{df}=5(\mathrm{P}<0.00001) ; \mathrm{I}^{2}=88 \%$} \\
\hline \multicolumn{14}{|c|}{ Test for overall effect: $Z=7.10(P<0.00001)$} \\
\hline Total $(95 \% \mathrm{Cl})$ & & & 394 & & & 485 & $100.0 \%$ & $4.26[2.81,5.71]$ & & & & & \\
\hline \multirow{2}{*}{\multicolumn{10}{|c|}{$\begin{array}{l}\text { Heterogeneity: } \mathrm{Tau}^{2}=4.11 ; \mathrm{Chi}^{2}=203.62, \mathrm{df}=7 \\
\text { Test for overall effect: } \mathrm{Z}=5.75(\mathrm{P}<0.00001)\end{array}$}} & -10 & -5 & 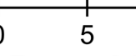 & 10 \\
\hline & & & & & & & & & & Favour & -sEGDV] & Favours & GDV] \\
\hline
\end{tabular}

Figure 2: Meta-analysis of the reduction of PVF.

\begin{tabular}{|c|c|c|c|c|c|c|c|c|c|}
\hline \multirow[b]{2}{*}{ Study or Subgroup } & \multicolumn{3}{|c|}{ sEGDV } & \multicolumn{3}{|c|}{ n-sEGDV } & \multicolumn{2}{|r|}{ Mean Difference } & \multirow{2}{*}{$\begin{array}{c}\text { Mean Difference } \\
\text { IV. Random, } 95 \% \mathrm{Cl}\end{array}$} \\
\hline & Mean & SD & Total & Mean & SD & Total & Weight & IV, Random, $95 \% \mathrm{Cl}$ Year & \\
\hline Gong QH & 659.4 & 99 & 93 & 850.9 & 101.4 & 93 & $38.7 \%$ & $-191.50[-220.30,-162.70] 2013$ & $=$ \\
\hline Zhao $\mathrm{H}$ & 578.89 & 98.65 & 57 & 703.15 & 103.18 & 51 & $37.4 \%$ & $-124.26[-162.44,-86.08] 2011$ & 늡 \\
\hline Pan WN & 752.7 & 380.5 & 85 & 715.6 & 376.3 & 91 & $23.9 \%$ & $37.10[-74.80,149.00] 2009$ & \\
\hline Total $(95 \% \mathrm{Cl})$ & & & 235 & & & 235 & $100.0 \%$ & $-111.75[-197.13,-26.38]$ & \\
\hline \multicolumn{9}{|c|}{$\begin{array}{l}\text { Heterogeneity: } \mathrm{Tau}^{2}=4688.26 ; \mathrm{Chi}^{2}=19.87, \mathrm{df}=2(\mathrm{P}<0.0001) ; \mathrm{I}^{2}=90 \% \\
\text { Test for overall effect: } Z=2.57(\mathrm{P}=0.01)\end{array}$} & 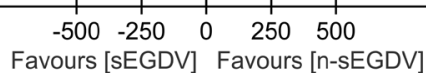 \\
\hline
\end{tabular}

Figure 3: Meta-analysis of portal vein flow.

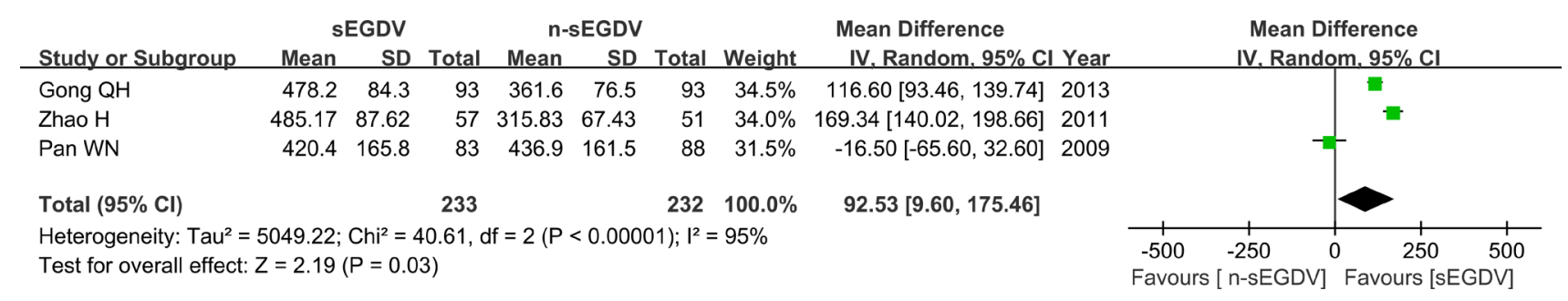

Figure 4: Meta-analysis of hepatic artery flow. 


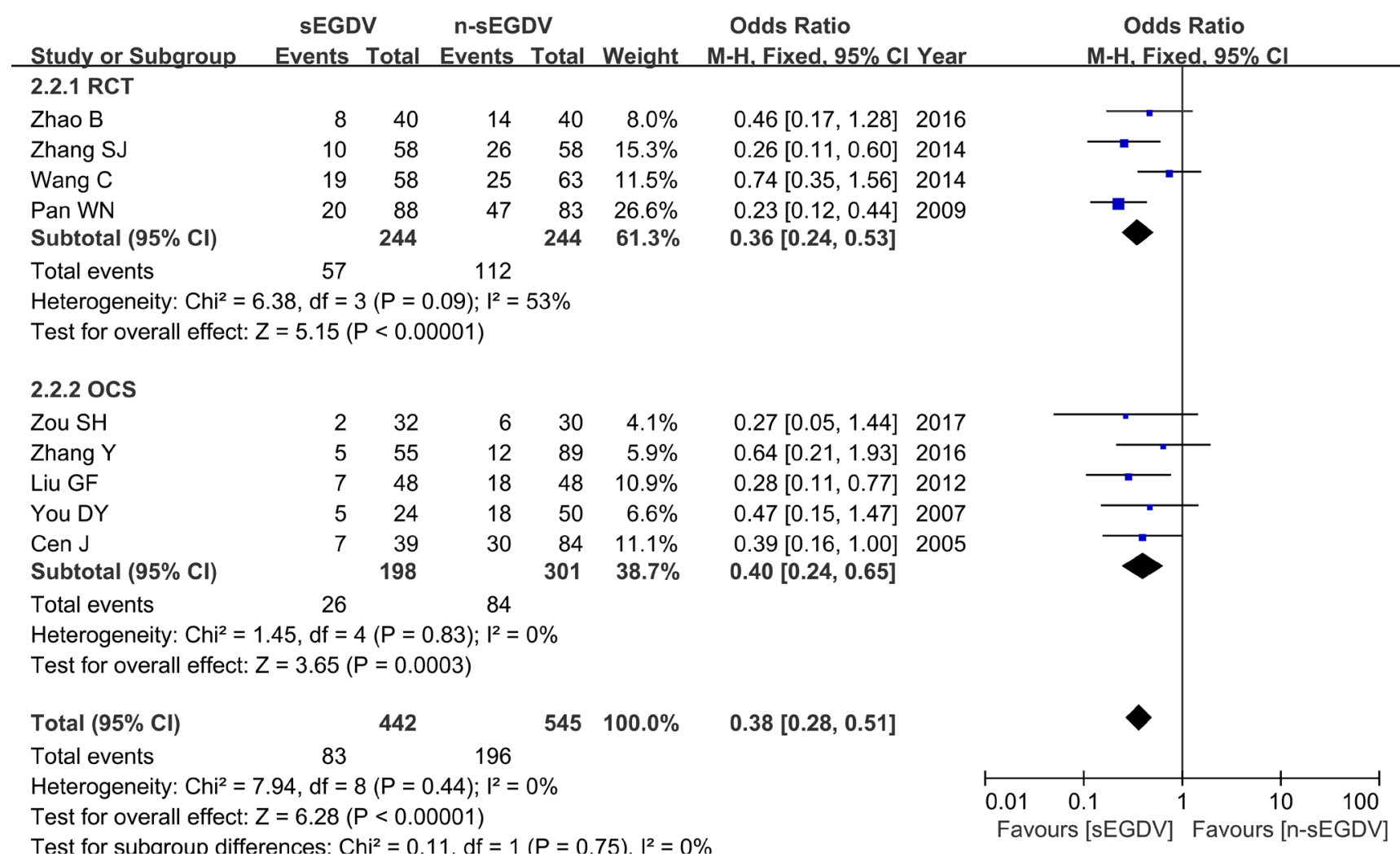

Figure 5: Meta-analysis of portal hypertensive gastropathy.

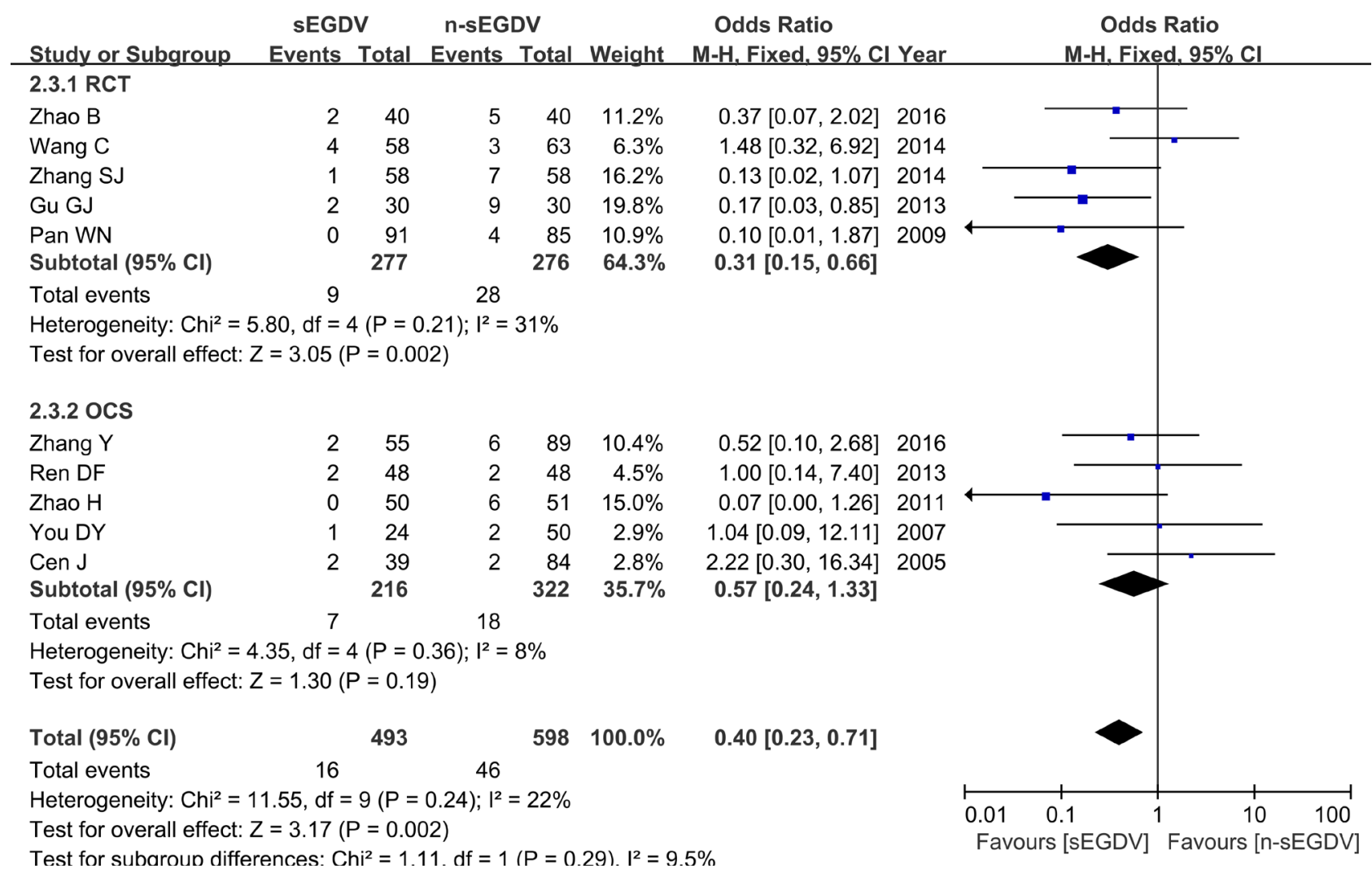

Figure 6: Meta-analysis of hepatic encephalopathy. 


\section{Postoperative mortality}

Eight included studies reported the postoperative mortality three year after operation. The results of metaanalysis show that there is significant difference between two groups in the incidence of postoperative mortality $\left(\mathrm{OR}=0.52 ; 95 \% \mathrm{CI}, 0.32-0.85 ; P=0.009 ; \mathrm{I}^{2}=0 \%\right.$ for heterogeneity), Therefore, using a Fixed model (Figure 8).

\section{Publication bias}

Deviation from this shape in Funnel plots can indicate publication bias. There was no evident asymmetry in the funnel plots (Figure 9), suggesting a low probability of publication bias.

\section{DISCUSSION}

Surgical treatment for liver cirrhosis complicated with portal hypertension mainly focuses on the prevention and treatment of esophageal varices bleeding, elimination of ascites, reduction of complications, and improvement in the overall quality of life [21]. An important factor to consider for evaluation of surgical approach for the treatment of portal hypertension is portal blood flow reduction as well as maintenance of hepatic blood inflow to prevent liver dysfunction and hepatic encephalopathy. There was a statistically significant difference in the PVF reduction between the two groups analyzed in this report. The results of our meta-analysis show that sEGDV was superior to n-sEGDV in reduction of PVF. Confirmed the effect of spontaneous portacaval shunt in decrease the PVF.

In recent years, many studies have shown that portal vein blood flow is reduced after devascularization, and that hepatic arterial blood flow increases as compensation to maintain the necessary blood supply to the liver [22]. In this study, the portal venous blood flow was reduced in both the sEGDV group and n-sEGDV group, and the postoperative blood flow of the portal vein in the SEGDV group was significantly lower than that in the n-sEGDV group. The postoperative arterial blood flow in the sEGDV and n-sEGDV groups all showed compensatory increase, and our meta-analysis results showed that the postoperative arterial blood flow of the portal vein in the sEGDV group was significantly higher than that in the n-sEGDV group. The mechanism is sEGDV group retained spontaneous shunt and portal vein blood flow decreased significantly, so the portal venous pressure and liver sinus pressure decreased significantly in the sEGDV group than in the n-sEGDV group. The hepatic artery velocity and flow showed an increase, while nitric oxide synthesis was inactivated and other vasodilator substances in the liver decreased due to the spontaneous shunt. These vasodilator substances resulted in the increase of hepatic

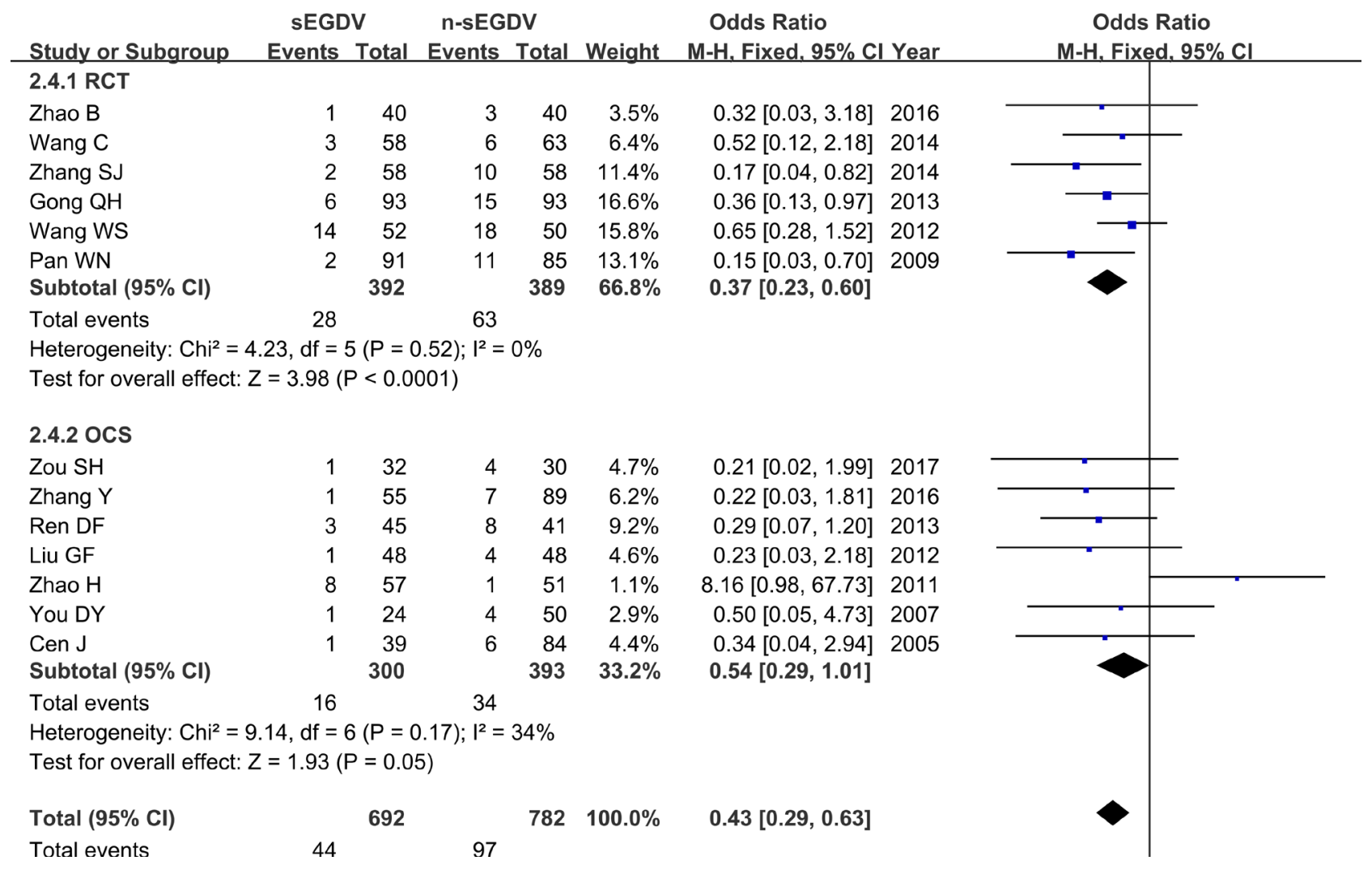

Figure 7: Meta-analysis of postoperative re-bleeding. 


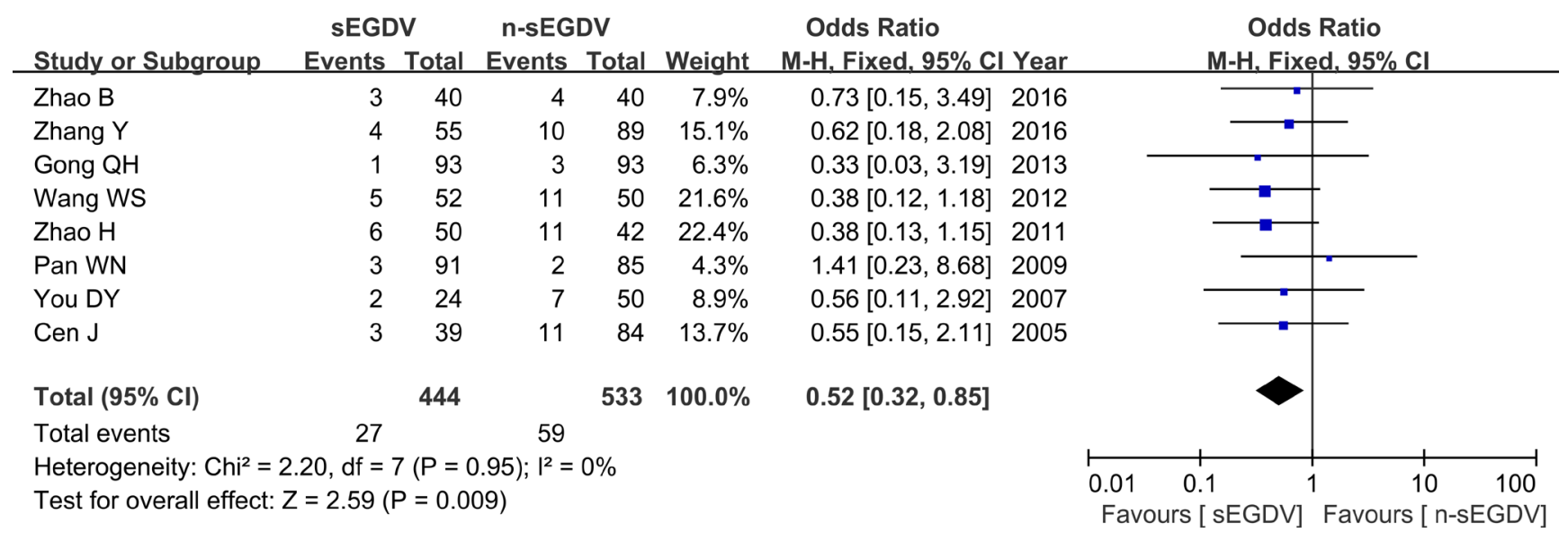

Figure 8: Meta-analysis of postoperative mortality.

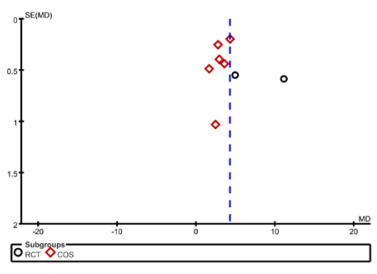

A
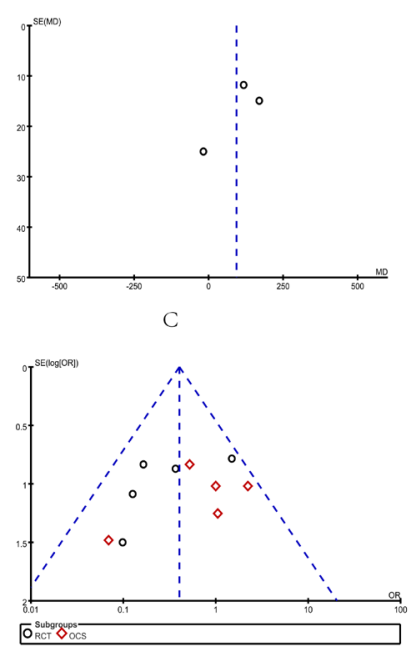

$\mathrm{E}$

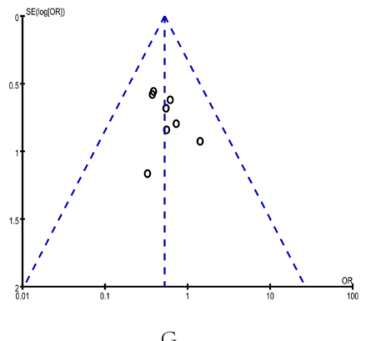

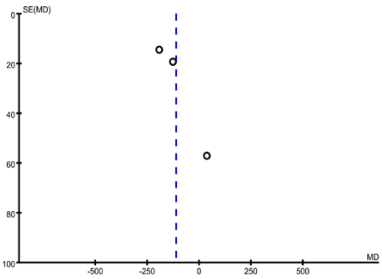

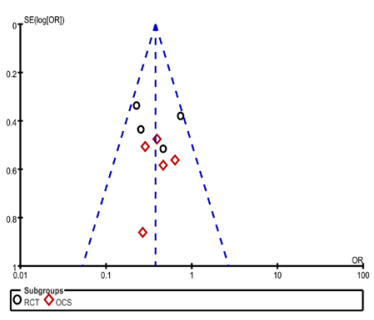

D

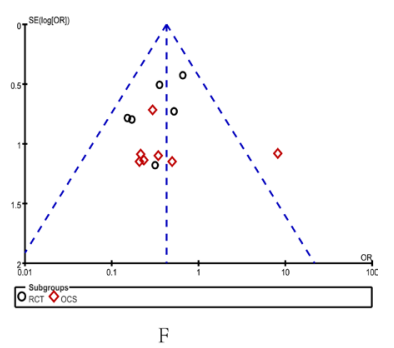

Figure 9: Funnel plots: Funnel plots were created to assess the publication bias in our meta-analysis of included studies. In the absence of publication bias, it assumes that studies with high precision will be plotted near the average, and studies with low precision will be spread evenly on both sides of the average, creating a roughly funnel-shaped distribution. (A) Reduction of PVF (B) Portal vein flow (C) Hepatic artery flow (D) Portal hypertensive gastropathy (E) Hepatic encephalopathy (F) Postoperative re-bleeding (G) Postoperative mortality 
artery blood flow and the ratio of hepatic artery in liver blood supply.

sEGDV preserves the continuous of portoazygous spontaneously shunt can alleviate visceral congestion and reduce the incidence of portal vein thrombosis and portal hypertensive gastropathy, thereby increasing the incidence of postoperative portal hypertension gastropathy [23-25]. This meta-analysis also confirmed that sEGDV was superior to n-sEGDV in reducing the rate of postoperative portal hypertension gastropathy. Compared to PSS, esophagogastric devascularization had no significant effect on the blood flow and function of the liver, which can prevent hepatic encephalopathy after operation. Our study showed that no significant difference existed between the sEGDV and n-sEGDV groups with respect to the rate of postoperative hepatic encephalopathy.

The reduction in the rate of re-bleeding resulting from moderation of hyperpressure of the portal vein should also be considered. Splenectomy significantly reduces the blood flow of the portal vein and decreases portal venous pressure. The traditional esophagogastric devascularization completely blocks the area around the esophagus and gastric fundus blood vessels and promotes the formation of new traffic branch veins of the portal vein and the azygos venous system, eventually leading to recurrence of esophageal varices and bleeding. Selective devascularization avoids the complete disconnection of shunt vessels and preserves the continuity of the portoazygous spontaneously shunt. Therefore, sEGDV can decrease the recurrence rate of varicose veins and rate of re-bleeding. The result of our meta-analysis also confirmed that sEGDV was superior to $\mathrm{n}$-sEGDV in reducing the rate of postoperative re-bleeding.

The postoperative mortality was significantly lower in the sEGDV group than in the n-sEGDV group [26-27]. This may be due to lower incidence of re-bleeding and hepatic encephalopathy in the sEGDV group. Thus, sEGDV was a more effective treatment with fewer complications for portal hypertension complicated with thoracic esophageal varices and bleeding than n-sEGDV. However, the paraesophageal veins cannot be preserved in every patient. If the vein trunk of the paraesophageal veins directly enter the esophageal wall, or it is difficult to identify the paraesophageal vein due to the varicose vessels from the venous plexus or bolus, then it should be devascularized from the beginning of the gastric coronary vein to ensure that the abnormal blood flow to the esophagogastric varices is blocked, to control and reduce the fatal variceal bleeding.

A major limitation of our meta-analysis is that it only included a small number of high-quality RCTs, all coming from the same country - China. Another potential limitation is that surgical experience and methods used at different hospitals and specialist centers could have produced different outcomes and increased the heterogeneity between the included studies. In addition, the treatment of complications may have affected the outcome of the RCTs and OCSs included in this meta-analysis.

\section{CONCLUSIONS}

sEGDV offers a more effective surgical approach with fewer complications to treat portal hypertension than n-sEGDV. Upon further detailed analysis of the surgical indications and hemodynamic and postoperative major complications of selective devascularization, sEGDV likely will provide us with a new direction in the choice of surgical approach for portal hypertension.

\section{CONFLICTS OF INTEREST}

The authors declare that there is no conflicts of interest regarding the publication of this paper.

\section{FUNDING SUPPORT}

This work was supported by CAMS Innovation Fund for Medical Sciences (CIFMS) grant number 2016I2M-1-001.

\section{REFERENCES}

1. Zhou GW, Li HW. Surgical therapy for portal hypertension in patients with cirrhosis in China: present situation and prospects. J Chin Med. 2009; 122:1483-1485.

2. Jiang HC, Sun B, Qiao HQ. Modified Sugiura procedure for the treatment of portal hypertension in 85 cases. Chin J Oper Proc Gen Surg. 2009; 3:20-22.

3. Corbett C, Mangat K, Olliff S. The role of transjugular intrahepatic portosystemic stent-shunt (TIPSS) in the management of variceal hemorrhage. Liver Int. 2012; 32:1493-1504.

4. Full D. Portal hypertension and varices in patients with liver cirrhosis. Nurs Stand. 2012; 26:52-57.

5. Zhang $\mathrm{N}, \mathrm{Yu} \mathrm{JH}, \mathrm{Xu}$ GL. Clinical application of the selective devascularization. J Hepatobiliary Surg. 2011; 19:235-237.

6. Yang Z. Anatomy naming and steps of selective periesophagogastric devascularization. J Surg Concepts Pract. 2009; 14:82-85.

7. Zhao B, Cheng QB. Clinical analysis of selective pericardial devascularization in the treatment of portal hypertension. Hennan journal of surgery. 2016; 22:11-13.

8. Zhang SJ. Comparison between selected splenectomy and sugiura operation on patients with portal hypertension. China Journal of Modern Medicine. 2014; 24:36-39.

9. Chao W, Liang X, Juan H, Chang J, Yin P, Zhen Y. A Prospective Randomized Trial of Selective versus Nonselective Esophagogastric Devascularization for 
Portal Hypertension. J Huazhong Univ Sci Technol. 2014; 34:563-568.

10. Gu GJ. Comparative study of selective pericardial devascularization and conventional devascularization in the treatment of portal hypertension. China Journal of Modern Medicine. 2013; 16:26-29.

11. Gong QH, Zhu SM, Huang X, Wang LB, Zhang CF. Clinical analysis of selective devascularization around portal vein in the treatment of cirrhotic portal hypertension. Chinese Journal of Medical Guide. 2013, 15:10-11.

12. Wang WS, Zou J, Li YK. Selective and non -selective pericardial blood vessel from five-year follow-up study of the efficacy of the off patients with portal hypertension. Chin J Curr Adv Gen Surg. 2012; 15:11-12.

13. Pan WN, Zhang QH, Zhang XF, Xu G, Ye Y. Comparative therapeutic effects of selective and non-selective devascularization procedures in the surgical treatment of portal hypertension. J Surg Concepts Pract. 2009; 14:23-25.

14. Zou SH, Xia XH, Li ZD. Effect analysis of splenectomy combined with selective pericardial devascularization in the treatment of portal hypertension. Chinese Journal of General Surgery. 2017; 26:801-805.

15. Zhang Y, Li DX, Jin JS, He S. Selective and non-selective pericardial devascularization for the treatment of portal hypertension. Henan medical research. 2016; 25:6-9.

16. Liu GF, Tian WJ, Jiang YC. The value of selective pericardial devascularization in the treatment of portal hypertension. Chin J Prim Med Pharm. 2013; 20:13-15.

17. Ren DF, Niu XQ, Zheng G, Shao LW, Gao ZX, Zhong JL. Comparison of selective and nonselective pericardial devascularization for portal hypertension and upper gastrointestinal hemorrhage: a report of 86 cases. J Clin Surg. 2013; 21:24-29.

18. You DY, Chen ML, Yang Z. Clinical analysis of selective pericardial devascularization in the treatment of portal hypertension. JC lin Surg. 2007; 15:12-15.
19. Zhao H, Li BJ, Long HG, Jiang HP. Selective and nonselective paraesophagogastric devascularization for the treatment of portal hypertension. Journal of practical medicine. 2011; 27:15-19.

20. Cen J, Yang Z, Zhou YG, Li T, Wang XB, Gao ZQ. The therapeutic effect of selective pericardial devascularization on portal hypertention. Fu Bu Wai Ke. 2005; 18:17-19.

21. Yang $Z$. The latest surgical treatment of portal hypertension. Jinan Shandong Science and Technology Publishing House. 2005:16:472-473.

22. McCormack TT, Sims J, Eyre-Brook I. Gastric lesions in portal hypertension: inflammatory gastritis or congestive gastropathy? Gut. 1985; 26:1226-1232.

23. Li SL, Li YC, Xu WL. Laparoscopic splenectomy and periesophagogastric devascularization with endoligature for portal hypertension in children. J Laparoendosc Adv Surg Tech A. 2009; 19:545-550.

24. Shi B, Yang Z, Wang X, Liu X. Selective periesophagogastric devascularization in portal hypertension: results of 56 patients. Hepatogastroenterology. 2009; 56:492-497.

25. Wu ZY. Hemodynamic study of portal hypertension. Hepatobiliary Pancreal Dis Int. 2007; 6:457-458.

26. Wang C, Yang Z, Xiao L, Qian J. Prevention of postoperative portal vein thrombosis in patients undergoing selective periesophagogastric devascularization combined with omentorenopex. J Surg Concepts Pract. 2009; 14:543-545.

27. Yang Z. Portal hypertension surgery atlas. Shen Yang: Liao Ning Science Technology Publishing House. 2006:15:198-223. 